\title{
Limits on Achievable Robustness Against Coprime Factor Uncertainty*
}

\author{
LAURA CHRISTIAN柿 and JIM FREUDENBERG $\|$
}

\begin{abstract}
Frequency domain methods for investigating inherent design limitations of linear feedback systems have been applied to study the effect these limitations have upon the design methodology of McFarlane and Glover.
\end{abstract}

Key Words-Feedback control; frequency response; robust control.

\begin{abstract}
We consider the problem of robustness optimization against normalized coprime factor uncertainty in single-input, single-output systems. We show that loop shapes known from classical analysis to be inconsistent with closed-loop robust stability will tend to have poor optimal robustness. Such loop shapes include those with a high crossover frequency relative to a nonminimum phase zero, a low crossover frequency relative to an unstable pole, or a rapid rolloff rate near gain crossover. Our results consist of a set of lower bounds on the optimal cost of the robustness optimization problem, each lower bound being appropriate to one of these three problematic loop shapes. The lower bounds are derived using the Poisson integral, and display the qualitative relationship between the loop shape and the level of optimal robustness.
\end{abstract}

\section{INTRODUCTION}

IN RECENT years, the problem of optimizing robustness in closed-loop systems against additive perturbations to the plant coprime factors has received much attention. Vidyasagar and Kimura (1986) showed that finding a robustly stabilizing controller for the above problem reduces to solving an $H_{\infty}$ optimization problem. Glover and McFarlane (1989) linked the $H_{\infty}$ optimization problem to the Nehari extension problem and, by using the normalized coprime factorization of the plant, derived a state-space solution that avoids the iterative methods usually required for $H_{\infty}$ controller synthesis. These authors introduce a design methodology in

\footnotetext{
* Received 9 February 1993; received in final form 3 January 1994. This paper was not presented at any IFAC meeting. This paper was recommended for publication in revised form by Associate Editor Hidenori Kimura under the direction of Editor Huibert Kwakernaak. Corresponding author Dr J. S. Freudenberg. Tel. +1313763 0586; Fax +1313 763 8041; E-mail jfr@zip.eecs.umich.edu.

† MS NP-2L, P.O. Box 800, Princeton NJ 08543, U.S.A.

$\ddagger$ Research performed at the University of Michigan, and supported by an NSF Graduate Fellowship.

\& EECS Department, University of Michigan, Ann Arbor MI 48109-2122, U.S.A.

\| Research supported by NSF Grant 8857510.
}

McFarlane and Glover (1990) that blends classical and multivariable loop shaping and robust optimization techniques. Georgiou and Smith (1990) show that optimizing robustness for normalized coprime factor uncertainty is equivalent to optimizing robustness in the gap metric.

Our work is motivated by the design methodology introduced in McFarlane and Glover (1990). Their design procedure combines loop shaping techniques from classical control with $H_{\infty}$ synthesis to optimize robustness against coprime factor uncertainty. Briefly, the design procedure consists of two stages, which we now summarize for a single-input, single-output plant. First, the Bode magnitude plot of the nominal plant is modified by a compensator to achieve a loop shape that reflects desired design goals, such as high gain at low frequencies for small sensitivity and low gain at high frequencies for small complementary sensitivity. This step is performed without regard to the plant phase, and hence without regard to nominal stability and gain/phase margins. We will call the result of this step the desired loop shape. Second, an $H_{\infty}$ optimal controller is synthesized for the shaped plant to achieve stabilization and to optimize robustness against coprime factor uncertainty. We will call the result of this step the achieved loop shape. In general, the achieved loop shape may deviate significantly from the desired loop shape. McFarlane and Glover (1990, pp. 106-118) interpret the optimal cost of the $H_{\infty}$ synthesis problem as an indicator of the compatibility of the desired loop shape with closed-loop stability requirements. The argument is as follows. First, they show that the discrepancy between the achieved and desired loop shapes at a given frequency is determined by the controller gain at that frequency. The controller gain, in turn, is bounded as a function of the optimal cost. If the optimal cost is small, 
then the achieved loop shape will be close to the desired loop shape. Since a large optimal cost corresponds to a small stability margin, the authors interpret the value of the optimal cost, and thus the deterioration in the desired loop shape, as an indication of the compatability of the loop shape with robust closed-loop stability.

In this paper, we study the relation between the value of the optimal cost and the compatibility of the desired loop shape with closed-loop stability. Restricting our attention to single-input, single-output systems allows us to study this problem using classical analysis techniques. From classical results, we know that certain loop shapes are incompatible with satisfactory closed-loop properties. These include loops with:

(i) a high crossover frequency relative to the location of a nonminimum phase zero;

(ii) a low crossover frequency relative to the location of an unstable pole; and

(iii) a rapid rolloff rate near gain crossover frequency.

To explore the relationship between the optimal cost and the desired loop shape, we will derive for a given desired loop shape, three lower bounds on the optimal cost. If any of these lower bounds is large then, as observed in McFarlane and Glover (1990), the achieved loop shape will deviate significantly from the desired loop shape. Hence, the desired loop shape is inconsistent with closed-loop robust stability requirements. These bounds are useful in identifying the reasons why the desired loop shape is incompatible with closed-loop stability, and they give insight into tradeoffs between conflicting design goals.

We emphasize that the utility of our bounds lies in the proof they provide that certain loop shapes are incompatible with closed-loop robust stability and for the qualitative insight they provide into the nature of the incompatibility. By way of contrast, our bounds are more difficult to evaluate than the optimal cost itself; however, the optimal cost does not display the qualitative information contained in the bounds.

In Section 2, we introduce our notation and describe the design methodology of McFarlane and Glover (1990). In Sections 3-5, we derive the lower bounds on the optimal cost for loop shapes that satisfy each of the conditions (i)-(iii).

An abbreviated version of this paper appeared in Christian and Freudenberg (1993).

\section{LOOP SHAPING DESIGN PROCEDURE}

We consider a single-input, single-output (SISO) finite-dimensional, linear, time-invariant plant. Denote the transfer function of the plant by $G(s)$, and let $[A, B, C, D]$ be a minimal realization of $G(s)$. Let $K(s)$ denote the compensator transfer function.* Define the sensitivity function, $S(s):=(1+G(s) K(s))^{-1}$ and the complementary sensitivity function, $T(s):=$ $G(s) K(s)(1+G(s) K(s))^{-1}$.

We will consider a normalized coprime factorization of the plant (McFarlane and Glover, 1990), $G(s)=M(s)^{-1} N(s)$, where $N(s)$, $M(s) \in H_{\infty}$ are coprime and satisfy the normalization condition $|N(j \omega)|^{2}+|M(j \omega)|^{2}=1$. Suppose that the coprime factors of the true plant are perturbed versions of those of the nominal plant:

$$
G_{\Delta}(s)=\left(M(s)+\Delta_{M}(s)\right)^{-1}\left(N(s)+\Delta_{N}(s)\right),
$$

where the uncertainty matrix $\Delta(s):=$ $\left[\Delta_{N}(s) \Delta_{M}(s)\right]$ satisfies $\Delta(s) \in R H_{\infty}$ and $\|\Delta\|_{\infty}<\varepsilon$ (McFarlane and Glover, 1990, pp. 29, 51). The robustness optimization problem we consider is to design a stabilizing controller $K(s)$ that maximizes the amount of coprime factor uncertainty the nominal system can tolerate without going unstable. As explained in McFarlane and Glover (1990, p. 52), this optimization problem is equivalent to that of minimizing the $H_{\infty}$ norm of the mixed sensitivity function $\dagger$

$$
S_{\text {mixed }}:=\left[\begin{array}{c}
K(1+G K)^{-1} M^{-1} \\
(1+G K)^{-1} M^{-1}
\end{array}\right]=\left[\begin{array}{c}
K S M^{-1} \\
S M^{-1}
\end{array}\right]
$$

Lemma 2.1 (McFarlane and Glover, 1990). The system $G_{\Delta}=\left(M+\Delta_{M}\right)^{-1}\left(N+\Delta_{N}\right)$ is stabilized by the controller $K$ for all $\|\Delta\|_{\infty}<\varepsilon$ if and only if

(i) $K$ stabilizes $G$, and

(ii) $\gamma:=\left\|S_{\text {mixed }}\right\|_{\infty}<1 / \varepsilon$.

Moreover, the maximum level of uncertainty that can be tolerated is given by $\varepsilon_{\max }=1 / \gamma_{\mathrm{opt}}$, where

$$
\gamma_{\mathrm{opt}}=\inf _{K \text { stab }}\left\|S_{\text {mixed }}\right\|_{\infty}
$$

The value of $\gamma_{\text {opt }}$ can be calculated explicitly as follows:

Lemma 2.2 (McFarlane and Glover, 1990, pp. $54-55,63)$. Let $G=[A, B, C, D]$. Then $\gamma_{\text {opt }}=$ $\sqrt{1+\lambda_{\max }(Z X)}$, where $X$ and $Z$ are the unique

\footnotetext{
*We adopt a negative feedback convention, rather than the positive feedback convention used in McFarlane and Glover (1990).

tWe suppress dependency upon the frequency variable when convenient.
} 
positive definite solutions to the Generalized Control Algebraic Riccati Equation (GCARE)

$$
\begin{aligned}
\left(A-B Q^{-1} D^{\mathrm{T}} C\right)^{\mathrm{T}} X & +X\left(A-B Q^{-1} D^{\mathrm{T}} C\right) \\
& -X B Q^{-1} B^{\mathrm{T}} X+C^{\mathrm{T}} R^{-1} C=0
\end{aligned}
$$

and to the Generalized Filtering Algebraic Riccati Equation (GFARE)

$$
\begin{aligned}
\left(A-B D^{\mathrm{T}} R^{-1} C\right) Z+ & Z\left(A-B D^{\mathrm{T}} R^{-1} C\right)^{\mathrm{T}} \\
& -Z C^{\mathrm{T}} R^{-1} C Z+B Q^{-1} B^{\mathrm{T}}=0,
\end{aligned}
$$

respectively, with $Q:=1+D^{2}$ and $R:=1+D^{2}$.

Note that $\gamma_{\mathrm{opt}}$ is bounded below by one. For later reference, we note that $\gamma_{\text {opt }}$ is invariant under a constant $180^{\circ}$ change in the phase of $G(s)$.

Lemma 2.3. Consider $G:=[A, B, C, D]$. Then

$$
\gamma_{\text {opt }}(G)=\gamma_{\text {opt }}(-G) \text {. }
$$

Proof. Follows immediately from an inspection of GCARE and GFARE.

We now describe the loop shaping design procedure of McFarlane and Glover (1990) in the special case of a SISO system.

\subsection{The loop shaping design procedure}

(McFarlane and Glover, 1990, p. 106)

(1) Loop shaping - use a precompensator, $W$, to shape the gain of the nominal plant to obtain a desired open-loop shape. Combine the nominal plant, $G$, and the compensator, $W$, to form the shaped plant, $G_{\mathrm{S}}$, where $G_{\mathrm{s}}:=W G$ and has no hidden unstable modes.

(2) Robust stabilization-calculate $\varepsilon_{\max }$. If $\varepsilon_{\max } \ll 1$, then return to (1) and redefine $W$. Otherwise, select $\varepsilon \leq \varepsilon_{\max }$ and use $H_{\infty}$ synthesis to generate a feedback controller, $K_{\infty}$, which robustly stabilizes the normalized coprime factorization of $G_{S}$ against coprime factor uncertainty satisfying $\|\Delta\|_{\infty}<\varepsilon$.

(3) Controller design-the final feedback controller, $K$, is obtained by combining the $H_{\infty}$ controller, $K_{\infty}$, and the shaping function, $W$, yielding $K=W K_{\infty}$.

Note that the role of the weighting functions used in the usual $H_{\infty}$ synthesis procedure is taken by the precompensator used to shape the plant magnitude to achieve the desired loop shape. Typically, the desired loop shape will have high gain at low frequencies to achieve small sensitivity, and low gain at high frequencies to achieve small complementary sensitivity.

In the sequel we shall assume with no loss of generality that $W(s)=1$. Results for the case of nonidentity weightings may be obtained by replacing $G(s)$ with $G_{\mathrm{S}}(s)$ in the relevant formulas.

For this design procedure to be effective, one must understand the relation between the desired loop shape and the resulting value of $\gamma_{\text {opr }}$. Such understanding will be useful in choosing a desired loop shape that will yield a reasonable value of $\gamma_{\mathrm{opt}}$, either a priori, or via successive iteration. It is clear from the discussion in McFarlane and Glover (1990) that desired loop shapes having one of the characteristics (i)-(iii) listed in Section 1 should tend to yield a large value of $\gamma_{\text {opt }}$. However, no proof of this statement is presented. Our goal is to present such a proof for each of (i)-(iii).

To derive our bounds, we shall utilize the following result, which is a straightforward consequence of the Poisson integral; for a proof see Freudenberg and Looze (1985).

Lemma 2.4. (Poisson Integral formula). Let $f(s)$ be rational, analytic, and nonzero in the closed right half-plane (CRHP) except for possible zeros on the imaginary axis. Then the value of $\log f(s)$ at any point $s=x+j y, \quad x>0$, is determined completely from the values of $\log f(j \omega), \omega \in \mathbf{R}$ :

$$
\log f(s)=\frac{1}{\pi} \int_{-\infty}^{\infty} \log f(j \omega) \frac{x}{x^{2}+(y-\omega)^{2}} \mathrm{~d} \omega .
$$

We will factor a nonminimum phase, unstable transfer function into a minimum phase, stable portion and two Blaschke products, defined below.

Definition 2.5 (Doyle et al., 1992, pp. 94-95; Freudenberg and Looze, 1988, pp. 32-36). Consider a rational transfer function $G(s)$ with poles and zeros in the open right half-plane (ORHP) $\left\{z_{i}, i=1, \ldots, N_{z}\right\}$ and $\left\{p_{i}, j=1, \ldots, N_{\mathrm{p}}\right\}$. Define the Blaschke products of nonminimum phase zeros

$$
B_{z}(s):=\prod_{i=1}^{N_{z}} \frac{z_{i}-s}{z_{i}+s}
$$

and unstable poles

$$
B_{\mathrm{p}}(s):=\prod_{j=1}^{N_{0}} \frac{p_{j}-s}{p_{j}+s} .
$$

Then

$$
G(s)=G_{0}(s) B_{\mathrm{z}}(s) B_{\mathrm{p}}^{-1}(s),
$$


where $G_{0}(s)$ has no poles or zeros in the open right half-plane.

For later reference, note that each Blaschke product is allpass of unit magnitude, and thus can be viewed as contributing additional phase lag (for a zero) or phase lead (for a pole) without changing the gain of $G(s)$.

\section{NONMINIMUM PHASE PLANTS}

A standard feedback problem requires disturbance rejection over a low frequency range. This requirement is stated as an upper bound on sensitivity at low frequencies. Equivalently, the requirement imposes a lower bound that the open-loop gain must satisfy over this frequency range. It is known that nonminimum phase plants pose a potential difficulty in robustly achieving such a design specification. Specifically, for such plants, a loop shape achieving small sensitivity (large open-loop gain) throughout a low frequency range can be obtained only at the expense of large sensitivity at other frequencies. Since large sensitivity corresponds to the Nyquist plot being close to the critical point, the stability margin of the resulting feedback system will be small. Hence, good feedback properties at low frequencies are obtained at the expense of poor properties at higher frequencies. The location of the nonminimum phase plant zeros relative to the region of small sensitivity plays a crucial role in this tradeoff. Specifically, when the region of small sensitivity extends into the frequency range where the zero contributes significant phase lag, the peak in sensitivity will be larger than when the zero is located outside this region (Freudenberg and Looze, 1988, pp. 31-44).

Since the design tradeoff just described is inherent in linear time-invariant feedback systems, it must manifest itself in the design procedure of McFarlane and Glover (1990). Indeed, the authors of McFarlane and Glover (1990) present an example of a nonminimum phase plant and show for this example that the size of the optimal cost grows as the open-loop crossover frequency is increased relative to the location of the zero. We shall now derive a lower bound on $\gamma_{\text {opt }}$ illustrating the relation between the desired loop shape, the nonminimum phase zero location, and the value of the optimal cost.

Theorem 3.1. Let $G(s)$ be a scalar rational transfer function. Suppose that $G(s)$ has a zero, $z$, in the open right half-plane. Then

$$
\gamma_{\text {opt }} \geq \gamma_{\text {lower bound }}
$$

where

$$
\begin{array}{r}
\log \left(\gamma_{\text {lower bound }}\right) \\
:=\frac{1}{2 \pi} \int_{0}^{\infty} \log \left(1+|G(j \omega)|^{2}\right) W(z, \omega) \mathrm{d} \omega \\
-\log \left|B_{\mathrm{p}}(z)\right| .
\end{array}
$$

For real $z=x$,

$$
W(z, \omega)=\frac{2 x}{x^{2}+\omega^{2}}
$$

and for complex $z=x+j y$

$$
W(z, \omega)=\frac{x}{x^{2}+(y-\omega)^{2}}+\frac{x}{x^{2}+(y+\omega)^{2}} .
$$

Proof. Internal stability requires that the optimal sensitivity function satisfy $S(z)=1$ at each zero of $G(s)$ in the open right half-plane. It follows from (1) and (2) and the maximum modulus theorem that the optimal sensitivity function satisfies $\gamma_{\text {opt }} \geq\left|S(s) M^{-1}(s)\right|, \quad \forall s \in$ CRHP, and thus that

$$
\gamma_{\mathrm{opt}} \geq\left|M^{-1}(z)\right| \text {. }
$$

Next, factor $M(s)=M_{0}(s) B_{\mathrm{p}}(s)$, where $M_{0}(s)$ has no zeros in the ORHP and $B_{\mathrm{p}}(s)$ is the Blaschke product of ORHP poles of $G(s)$. Applying the Poisson Integral formula to $M_{0}(s)$ yields

$$
\begin{aligned}
& \log \left|M_{0}^{-1}(z)\right|=\frac{1}{\pi} \int_{0}^{\infty} \log \left|M_{0}^{-1}(j \omega)\right| W(z, \omega) \mathrm{d} \omega \\
& \text { or, since }\left|M^{-1}(j \omega)\right|=\left|M_{0}^{-1}(j \omega)\right| \text { and } M^{-1}(z)= \\
& \begin{aligned}
M_{0}^{-1}(z) B_{\mathrm{p}}^{-1}(z): & \\
\log \left|M^{-1}(z)\right|= & \frac{1}{\pi} \int_{0}^{\infty} \log \left|M^{-1}(j \omega)\right| W(z, \omega) \mathrm{d} \omega \\
& +\log \left|B_{\mathrm{p}}^{-1}(z)\right| .
\end{aligned}
\end{aligned}
$$

It is shown in McFarlane and Glover (1990, pp. 127-128) that $|M(j \omega)|=\frac{1}{\sqrt{1+|G(j \omega)|^{2}}}$. Hence,

$$
\begin{aligned}
\log \left|M^{-1}(z)\right| & \\
=\frac{1}{\pi} \int_{0}^{\infty} \log \sqrt{1+|G(j \omega)|^{2}} & W(z, \omega) \mathrm{d} \omega \\
& +\log \left|B_{\mathrm{p}}^{-1}(z)\right| .
\end{aligned}
$$

Together, (8) and (9) yield (7).

For a given loop shape $|G(j \omega)|$, the lower bound (7) may be evaluated directly via numerical integration. It is also instructive to consider an estimate for the bound.

Corollary 3.2. Let $G(s)$ be a scalar rational transfer function, with a zero, $z$, in the open 
right half-plane. Suppose that

$$
|G(j \omega)| \geq \alpha>1, \quad \forall \omega \leq \omega_{1} .
$$

Then

$$
\gamma_{\mathrm{opt}} \geq\left(1+\alpha^{2}\right)^{W(z, \Omega) / 2 \pi}\left|B_{\mathrm{p}}^{-1}(z)\right|,
$$

where $\Omega:=\left[0, \omega_{1}\right], W(z, \Omega):=\int_{0}^{\omega_{1}} W(z, \omega) \mathrm{d} \omega$.

Proof. From Theorem 3.1,

$$
\begin{aligned}
& \log \left(\gamma_{\mathrm{opt}}\right) \\
& \geq \frac{1}{\pi} \int_{0}^{\infty} \log \left(1+|G(j \omega)|^{2}\right) W(z, \omega) \mathrm{d} \omega-\log \left|B_{\mathrm{p}}(z)\right| \\
& \geq \frac{1}{2 \pi} \int_{0}^{\omega_{1}} \log \left(1+|G(j \omega)|^{2}\right) W(z, w) \mathrm{d} \omega-\log \left|B_{\mathrm{p}}(z)\right| \\
& \geq \frac{1}{2 \pi} \log \left(1+\alpha^{2}\right) \int_{0}^{\omega_{1}} W(z, \omega) \mathrm{d} \omega-\log \left|B_{\mathrm{p}}(z)\right| .
\end{aligned}
$$

Taking the inverse log yields (10).

Theorem 3.1 and Corollary 3.2 show that the lower bound on $\log \left(\gamma_{\text {opt }}\right)$ will be large (i) if $G(s)$ has an unstable pole near a nonminimum phase zero, or (ii) if $|G(j \omega)|$ is large over a frequency range that is wide relative to the location of the nonminimum phase zero. Let us elaborate upon the latter condition. In Freudenberg and Looze (1988), the authors show that for real $z=x$, $W(z, \Omega)=-\angle \frac{x-j \omega_{1}}{x+j \omega_{1}}$, the negative of the phase lag contributed by the term due to the zero in the factorization of the plant (6). It follows that $\gamma_{\text {opt }}$ will be large if the region of high gain extends to frequencies at which the zero contributes significant additional phase lag. A similar interpretation holds for complex zeros.

In McFarlane and Glover (1990, pp. 108-109) an example of a nonminimum phase plant is used to illustrate that the optimal cost increases as the region of large gain extends to high frequency relative to the nonminimum phase zero. We use this example to compare the results of our bound and the value of the optimal cost.

Example 3.3. Let $G(s)=\frac{k(-1+s)}{s(s+2)}$, where $k$ varies from 0.1 to 20 to vary the open-loop gain crossover frequency. Applying Lemma 2.2 and Theorem 3.1, we calculate $\gamma_{\text {opt }}$ and $\gamma_{\text {lower bound }}$ for various values of $k$. The first term in (7) was evaluated via numerical integration. Table 1 shows that as the crossover frequency increases both $\gamma_{\text {opt }}$ and the lower bound $\gamma_{\text {lower bound }}$ become large. These results are consistent with those of McFarlane and Glover (1990).

Alternately, it is instructive to fix the crossover frequency and vary the location of the zero.

Example 3.4. Let $G(s)=\frac{30}{s(s+0.2)} \frac{(z-s)}{(z+s)}$, where $z$ varies from 0.01 to 100 . Applying Lemma 2.2 and Theorem 3.1, we calculate $\gamma_{\mathrm{opt}}$ and $\gamma_{\text {lower bound }}$ for different values of $z$. Figure 1 shows that $\gamma_{\text {opt }}$ and $\gamma_{\text {lower bound }}$ both become large as the zero decreases relative to the open-loop gain crossover frequency of $5 \mathrm{rad} \mathrm{s}^{-1}$.

The results of this section show that the well-known design limitations and tradeoffs due to nonminimum phase zeros manifest themselves in the loop shaping design procedure of McFarlane and Glover (1990).

\section{UNSTABLE PLANTS}

When a system to be controlled is open-loop unstable, classical design rules indicate that the loop gain should be shaped so that the gain crossover frequency is at least as large as the radius of the unstable poles (cf. Looze and Freudenberg, 1991). Since realistic feedback problems also include bandwidth constraints, this fact may lead to design conflicts. Specifically, when complementary sensitivity (and thus open-loop gain) is required to be small at frequencies that are relatively low with respect to the radius of the unstable poles, then there is necessarily a large peak in complementary sensitivity at lower frequencies. Mathematically, this phenomenon is dual to the tradeoff associated with nonminimum phase zeros described in Section 3. We shall now derive a lower bound on $\gamma_{\text {opt }}$ showing that if the gain of the desired loop shape is too low with respect to the pole location, then the value of the optimal cost will be large.

Theorem 4.1. Let $G(s)$ be a scalar rational transfer function. Suppose that $G(s)$ has a pole, $p$, in the open right half-plane. Then

$$
\gamma_{\text {opt }} \geq \gamma_{\text {lower bound }}
$$

TABLE 1. EFFECT OF VARYING CROSSOVER FREQUENCY

\begin{tabular}{rccc}
\hline$k$ & $\omega_{0}\left(\mathrm{rad} \mathrm{s}^{-1}\right)$ & $\gamma_{\text {opt }}$ & $\gamma_{\text {lower bound }}$ \\
\hline 0.1 & 0.05 & 1.4676 & 1.0486 \\
1.0 & 0.55 & 1.9850 & 1.5446 \\
5.0 & 4.5 & 4.5945 & 4.0697 \\
20.0 & 20.0 & 14.627 & 13.9785 \\
\hline
\end{tabular}




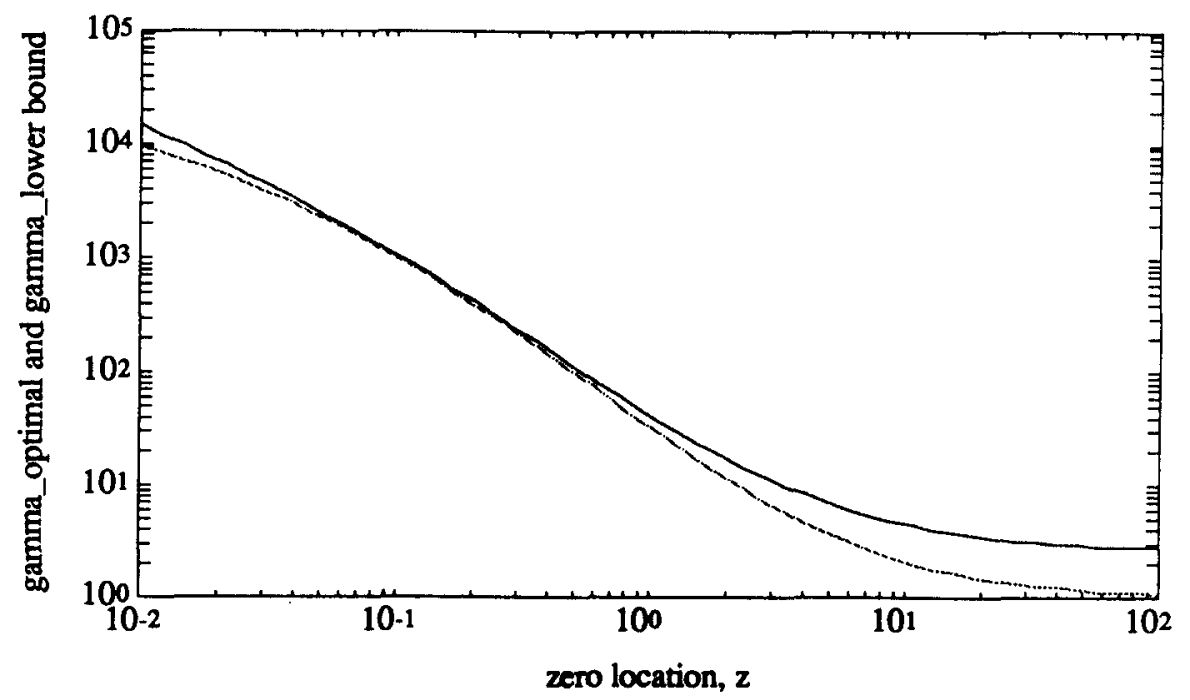

FIG. 1. $\gamma_{\mathrm{opt}}$ and $\gamma_{\text {lower bound }}$ vs zero location.

where

$\log \left(\gamma_{\text {lower bound }}\right)$

$$
\begin{array}{r}
=\frac{1}{2 \pi} \int_{0}^{\infty} \log \left(1+\frac{1}{|G(j \omega)|^{2}}\right) W(p, \omega) \mathrm{d} \omega \\
-\log \left|B_{\mathbf{z}}(p)\right| .
\end{array}
$$
For real $p=x, W(p, \omega)=\frac{2 x}{x^{2}+\omega^{2}}$ and for
complex $p=x+j y:$

$$
W(p, \omega)=\frac{x}{x^{2}+(y-w)^{2}}+\frac{x}{x^{2}+(y+w)^{2}}
$$

Proof. Note from (1) that $S_{\text {mixed }}(s)=$ $\left[\begin{array}{c}K S M^{-1} \\ S M^{-1}\end{array}\right]=\left[\begin{array}{c}T N^{-1} \\ S M^{-1}\end{array}\right]$. The remainder of the proof is entirely dual to that of Theorem 3.1, with the roles of $S$ and $T$, and of $M$ and $N$, interchanged. For later reference, we note that (9) is replaced by

$$
\begin{array}{r}
\log \left|N^{-1}(p)\right| \\
=\frac{1}{\pi} \int_{0}^{\infty} \log \sqrt{1+\frac{1}{|G(j \omega)|^{2}}} W(p, \omega) \mathrm{d} \omega \\
+\log \left|B_{z}^{-1}(p)\right| .
\end{array}
$$

Corollary 4.2. Let $G(s)$ be a scalar rational transfer function with a pole, $p$, in the open right half-plane. Suppose that

$$
|G(j \omega)| \leq \beta<1, \quad \forall \omega \geq \omega_{2} .
$$

Then

$$
\gamma_{\mathrm{opt}} \geq\left(1+\frac{1}{\beta^{2}}\right)^{W\left(p, \Omega^{\alpha}\right) / 2 \pi}\left|B_{\mathrm{z}}^{-1}(p)\right|,
$$

where $\quad \Omega^{\mathrm{c}}:=\left[\omega_{2}, \infty\right) \quad$ and $\quad W\left(p, \Omega^{\mathrm{c}}\right):=$ $\int_{\omega_{2}}^{\infty} W(p, \omega) \mathrm{d} \omega$.

Proof. Dual to that of Corollary 3.2 .

Theorem 4.2 shows that $\gamma_{\text {opt }}$ will be large (i) if $G(s)$ has a nonminimum phase zero near the unstable pole, or (ii) if $|G(j \omega)|$ is small above a frequency that is relatively low with respect to the location of the pole. Let us elaborate upon the latter condition. For real $z=x, W\left(p, \Omega^{\mathrm{c}}\right)=$ $\pi-\angle \frac{x+j \omega_{2}}{x-j \omega_{2}}$, or $\pi$ minus the additional phase lead contributed by the pole in the plant factorization (6). It follows that $\gamma_{\mathrm{opt}}$ will be large if $\omega_{2}$ in (13) is a frequency at which the additional phase lead contributed by the pole is negligible. This phenomenon is plausible because additional lead is needed to obtain the proper number of encirclements of the critical point needed for closed-loop stability.

\section{RAPID RATE OF ROLLOFF AT GAIN CROSSOVER}

In Section 3 we saw that achieving a loop shaping goal of high gain over a wide low frequency range for a nonminimum phase plant may be inconsistent with robust closed-loop stability. In Section 4 we saw a similar result for the problem of achieving a bandwidth constraint for an unstable plant. In each case, the existence of the limitation is due to the presence of a plant singularity that cannot be removed without violating internal stability. The analysis in each 
case was performed rather easily using the Poisson integral. The next design limitation we discuss is more subtle, and, as we shall see, requires more work to analyze.

Suppose that we desire high gain to achieve small sensitivity over a low frequency range, and low gain to achieve small complementary sensitivity over a high frequency range. Satisfying both these goals will require the loop transfer function to rolloff at a certain rate near the gain crossover frequency. The Bode gain/phase relation (cf. Freudenberg and Looze, 1988; Doyle et al., 1992) states that, for a stable minimum phase rational function, a $20 \mathrm{~N} \mathrm{db} /$ decade rate of gain decrease near crossover frequency will result in $-90 \mathrm{~N}^{\circ}$ phase lag at crossover. Hence if the gain decreases too rapidly, the Nyquist plot will either violate the encirclement count needed for closed-loop stability, or will have a poor phase margin. It follows that a design tradeoff exists between achieving high gain at low frequencies and low gain at high frequencies. This tradeoff is dictated by the need to achieve closed-loop stability as well as reasonable feedback properties at intermediate frequencies. The tradeoff becomes more problematic as the width of the intermediate frequency range narrows. An alternate means of analyzing this tradeoff is via the Bode sensitivity integral (cf. Freudenberg and Looze, 1988; Doyle et al., 1992).

The design limitations just described must manifest themselves in the loop shaping design procedure. We now show that if the shaped plant has either a poor phase margin or Nyquist encirclements inconsistent with closed-loop stability (both of which can occur due to excessively rapid rolloff near gain crossover), then the optimal cost will be large.

Theorem 5.1. Let $G(s)$ be a rational function with no poles or zeros in the open right halfplane. Define $G(0+):=\operatorname{limit}_{x \rightarrow 0 ; x>0} G(x)$ and suppose that $G(0+)>0$. Then, for all $x$ real and positive

with

$$
\gamma_{\text {opt }} \geq \gamma_{\text {lower bound }}(x)
$$

$$
\begin{aligned}
\log \left(\gamma_{\text {lower bound }}(x)\right) & \\
=\frac{1}{\pi} \int_{0}^{\infty} \log \frac{\sqrt{1+|G(j \omega)|^{2}}}{|1+G(j \omega)|} & W(x, \omega) \mathrm{d} \omega \\
& -\log \left|B_{\mathrm{p}}^{\prime}(x)\right|,
\end{aligned}
$$

where

(i) $B_{\mathrm{p}}^{\prime}(s)$ is the Blaschke product containing the unstable poles (if any) of $(1+G(s))^{-1}$ and

(ii) $W(x, \omega)=\frac{2 x}{x^{2}+\omega^{2}}$.
Furthermore, any poles of $(1+G(s))^{-1}$ in the open right half-plane must be complex.

Proof. It follows from (1) and (2) and the maximum modulus theorem that at each $x>0$

$$
\begin{array}{r}
\log \left(\gamma_{\text {opt }}\right) \geq \max \{\log |S(x)|-\log |M(x)|, \\
\log |T(x)|-\log |N(x)|\},
\end{array}
$$

where $S(s)$ and $T(s)$ are the optimal sensitivity and complementary sensitivity functions. Substituting (9), (12), and the identity $S(x)+T(x)=1$ into (15) yields two lower bounds upon the optimal cost:

$$
\begin{aligned}
& \log \left(\gamma_{\text {opt }}\right) \geq \log |S(x)|+\phi_{1}, \\
& \log \left(\gamma_{\text {opt }}\right) \geq \log |1-S(x)|+\phi_{2},
\end{aligned}
$$

where

$$
\phi_{1}=\frac{1}{2 \pi} \int_{0}^{\infty} \log \left(1+|G(j \omega)|^{2}\right) W(x, \omega) \mathrm{d} \omega
$$

and

$\phi_{2}=\frac{1}{2 \pi} \int_{0}^{\infty} \log \left(1+1 /|G(j \omega)|^{2}\right) W(x, \omega) \mathrm{d} \omega$.

We next eliminate the dependence of the lower bounds (16) and (17) upon the optimal sensitivity function by finding a lower bound, independent of the value of $S(x)$, upon the maximum of (16) and (17). To do this, we find a particular value of $S(x)$ that makes these lower bounds equal, and then demonstrate that any other value of $S(x)$ having this property necessarily yields a larger common value for the bounds. To proceed, observe the plots of $\log |S(x)|$ and $\log |1-S(x)|$ vs $S(x)$ in Fig. 2 . Adding $\phi_{1}$ and $\phi_{2}$ to yield (16) and (17) merely shifts each plot up or down by a constant without changing its shape. Note that there always exists a value of $S(x) \in(0,1)$ that makes $(16)$ and $(17)$ equal. Furthermore, note that (16) is a monotonically increasing function of $S(x)$ over the interval $(0, \infty)$. Hence if $(16)$ and $(17)$ are made equal for a value of $S(x)>1$, then necessarily the common value of these bounds is larger than that obtained for the value of $S(x) \in(0,1)$. A similar remark applies to any value of $S(x)<0$ making the two bounds equal.

We now derive an expression for the value of $S(x) \in(0,1)$ that makes the lower bounds $(16)$ and (17) equal. Setting these expressions equal, rearranging, and applying the Poisson integral (3) yields:

$$
\log \frac{|S(x)|}{|1-S(x)|}=-\log |G(x)| .
$$




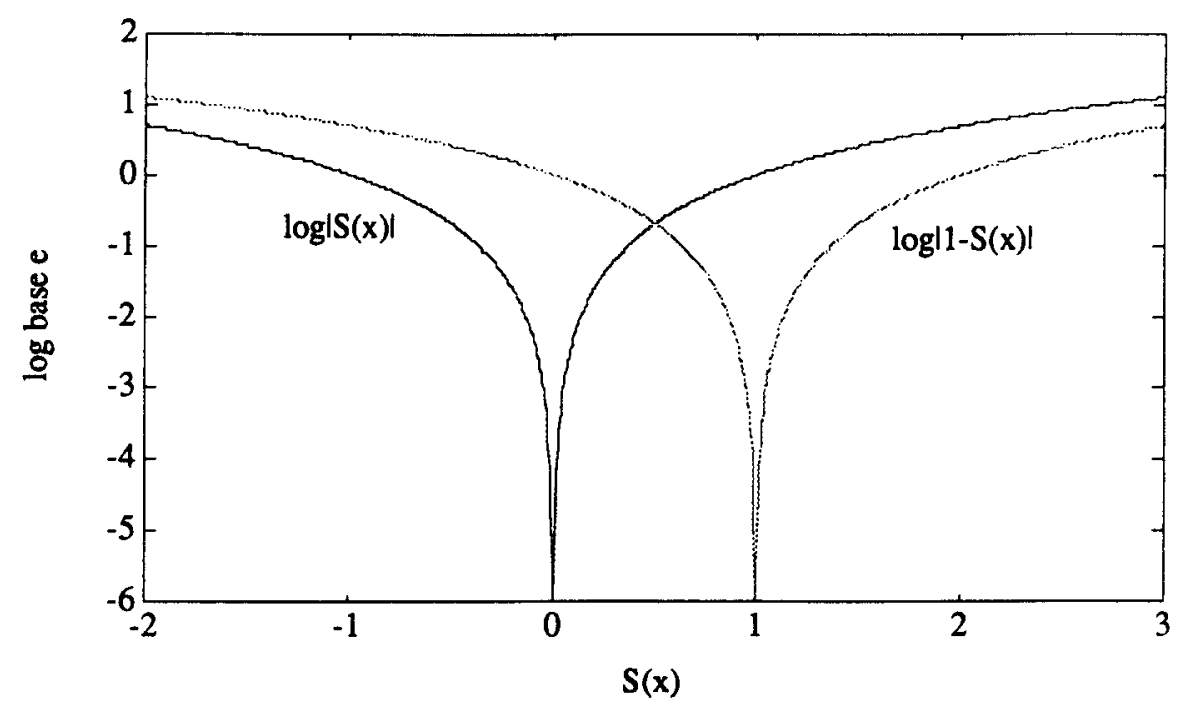

FIG. 2. $\log |S(x)|$ and $\log |1-S(x)|$ vs. $S(x)$.

Since $S(x) \in(0,1)$, it follows that the absolute value signs in (20) may be removed, yielding

$$
\log \frac{S(x)}{1-S(x)}=-\log |G(x)| .
$$

Solving for $S(x)$ in (21) yields

$$
S(x)=\frac{1}{|G(x)|+1} .
$$

Substituting (22) into (16) yields

$$
\begin{aligned}
\log \left(\gamma_{\mathrm{opt}}\right) & \geq \log \frac{1}{|G(x)|+1} \\
& +\frac{1}{2 \pi} \int_{0}^{\infty} \log \left(1+|G(j \omega)|^{2}\right) W(x, \omega) \mathrm{d} \omega .
\end{aligned}
$$

Since $G(s)$ has no poles or zeros in the open right half-plane and $G(0+)>0$ by assumption, it follows that $G(x)>0, \forall x>0$. Hence the absolute values can be removed from $|G(x)|$ in (23), yielding

$$
\begin{aligned}
& \log \left(\gamma_{\text {opt }}\right) \geq \log \left|S^{\prime}(x)\right| \\
& \quad+\frac{1}{2 \pi} \int_{0}^{\infty} \log \left(1+|G(j \omega)|^{2}\right) W(x, \omega) \mathrm{d} \omega,
\end{aligned}
$$

where

$$
S^{\prime}(s):=\frac{1}{(1+G(s))} .
$$

Factoring out the Blaschke product of unstable poles (if any) of $S^{\prime}(s)$ yields

$$
S^{\prime}(s)=S_{0}^{\prime}(s) B_{\mathrm{p}}^{\prime-1}(s),
$$

where $S_{0}^{\prime}(s)$ is stable and minimum phase and $B_{\mathrm{p}}^{\prime}(\mathrm{s})$ is the Blaschke product that contains the unstable poles of $S^{\prime}(s)$. Then, $\log \left(S_{0}^{\prime}(s)\right)$ is analytic in the open right half-plane, and the
Poisson Integral formula can be applied. This yields

$$
\log \left|S_{0}^{\prime}(x)\right|=\frac{1}{\pi} \int_{0}^{\infty} \log \left|S_{0}^{\prime}(j \omega)\right| W(x, \omega) \mathrm{d} \omega,
$$

or, since $\left|S_{0}^{\prime}(j \omega)\right|=\left|S^{\prime}(j \omega)\right| \quad$ and $\quad S^{\prime}(x)=$ $S_{0}^{\prime}(x) B_{\mathrm{p}}^{\prime-1}(x)$ :

$$
\begin{aligned}
\log \left|S^{\prime}(x)\right|= & \frac{1}{\pi} \int_{0}^{\infty} \log \left|S^{\prime}(j \omega)\right| W(x, \omega) \mathrm{d} \omega \\
& -\log \left|B_{\mathrm{p}}^{\prime}(x)\right|
\end{aligned}
$$

Substituting (28) into (24), using definition (25), and simplifying, yields (14). The second claim follows since, as we have argued, $G(x)>0$, for $x>0$, and thus $S^{\prime}(s)$ can have no real unstable poles.

Theorem 5.1 shows that $\gamma_{\mathrm{opt}}$ will tend to be large (i) if the Nyquist plot of $G(j \omega)$ is inconsistent with closed-loop stability, so that the second term in (14) is large, or (ii) if $G(j \omega)$ has a poor phase margin, so that the first term in (14) is large. [Note that since $(1+G(s))^{-1}$ can have no poles that are real and positive, the second term in (14) is finite.]

Theorem 5.1 is valid for any $x$, real and positive. To evaluate a bound on $\gamma_{\mathrm{opt}}$, we must choose a specific value of $x$. For the bound to be meaningful, the value of $x$ should tend to maximize at least one of the two terms on the right-hand side of (14). Consider the first term. If $G(j \omega)$ has a small phase margin, then the integrand will be large near the gain crossover frequency, which we shall denote $\omega_{\mathrm{c}}$. The value of the weighting function at this frequency, $W\left(x, \omega_{\mathrm{c}}\right)$, is maximized over $x$ by setting $x=\omega_{\mathrm{c}}$. Alternately, if $1 /(1+G(s))$ is unstable, the 
second term in (14) may be emphasized by setting $x$ equal to the radius of an unstable pole pair [recall our assumptions imply that $1 /(1+$ $G(s)$ ) can have no real unstable poles]. Often the radius of the unstable poles will be approximately equal to the gain crossover frequency. In the following example, we calculate $\gamma_{\text {lower bound }}$ by setting $x=\omega_{\mathrm{c}}$. The first term in (14) was evaluated via numerical integration.

Example 5.2. Let $G(s)=\frac{1}{s^{k}}$, where $k$ varies from 1 to 5. Using Lemma 2.2 and Theorem 5.1, we can calculate $\gamma_{\text {opt }}$ and $\gamma_{\text {lower bound }}$ for various values of $k$. The results are shown in Table 2. As $k$ increases, the rolloff rate increases, and $\gamma_{\text {opt }}$ and $\gamma_{\text {lower bound }}$ both become large.

Although the lower bound does not approximate $\gamma_{o p t}$ as closely as those in Sections 3 and 4 , it does indicate the trend of $\gamma_{\mathrm{opt}}$ and thus demonstrates the inherent problems in specifying a loop shape that requires rapid rolloff near crossover.

We now present a generalization of Theorem 5.1 to systems that have poles and/or zeros in the open right half-plane.

Theorem 5.3. Let $G(s)$ be a rational function. Consider $x$ real and positive, and suppose that $x$ is not a pole of $G(s)$. Then

$$
\gamma_{\text {opt }} \geq \gamma_{\text {lower bound }}(x)
$$

with

$$
\begin{array}{r}
\log \left(\gamma_{\text {lower bound }}(x)\right) \\
=\frac{1}{\pi} \int_{0}^{\infty} \log \frac{\sqrt{1+|G(j \omega)|^{2}}}{\left|1+G_{x}(j \omega)\right|} W(x, \omega) \mathrm{d} \omega \\
-\log \left|B_{\mathrm{p}}^{\prime}(x)\right|,
\end{array}
$$

where

$$
\text { (i) } G_{x}(s):=\left\{\begin{array}{rll}
G(s) & \text { if } & G(x) \geq 0 \\
-G(s) & \text { if } & G(x)<0
\end{array}\right. \text {, }
$$

(ii) $B_{\mathrm{p}}^{\prime}(s)$ is the Blaschke product containing the unstable poles (if any) of $\left(1+G_{x}(s)\right)^{-1}$, and

(iii) $W(x, \omega)=\frac{2 x}{x^{2}+\omega^{2}}$.

Furthermore, $\left(1+G_{x}(s)\right)^{-1}$ has no pole at $s=x$.

TABLE 2. EFFEct OF VARYING RATE OF GAIN DECREASE

\begin{tabular}{ccc}
\hline$k$ & $\gamma_{\text {opt }}$ & $\gamma_{\text {lower bound }}$ \\
\hline 1 & 1.4142 & 1.0000 \\
2 & 2.6131 & 1.6968 \\
3 & 5.9136 & 3.0001 \\
4 & 15.290 & 5.3202 \\
5 & 42.349 & 9.4662 \\
\hline
\end{tabular}

Proof. The proof follows that of Theorem 5.1 with the few exceptions noted below:

(i) In passing from (23) to (24), we used the fact that $G(x)>0$. Under present hypotheses, this need not be the case; hence we substitute $G_{x}(x)$ for $|G(x)|$ in making this transition. From Lemma 2.3, it follows that the optimal costs associated with $G(s)$ and $G_{x}(s)$ are equal in value. Hence we make this substitution with no loss of generality.

(ii) The expressions for $\phi_{1}$ and $\phi_{2}$ in (16) and (17) must be modified by the addition of terms due to the Blaschke products of open right half-plane poles and zeros of $G(s)$, respectively:

$$
\begin{aligned}
\phi_{1}= & \frac{1}{2 \pi} \int_{0}^{\infty} \log \left(1+|G(j \omega)|^{2}\right) W(x, \omega) \mathrm{d} \omega \\
& -\log \left|B_{\mathrm{p}}(x)\right|
\end{aligned}
$$

and

$$
\begin{aligned}
\phi_{2}= & \frac{1}{2 \pi_{0}} \int_{0}^{\infty} \log \left(1+1 /|G(j \omega)|^{2}\right) W(x, \omega) \mathrm{d} \omega \\
& -\log \left|B_{z}(x)\right| .
\end{aligned}
$$

It is straightforward to verify that (20) remains valid as stated. However, (23) and (24) become

$$
\begin{aligned}
\log \left(\gamma_{\mathrm{opt}}\right) \geq & \log \frac{1}{|G(x)|+1} \\
& +\frac{1}{2 \pi} \int_{0}^{\infty} \log \left(1+|G(j \omega)|^{2}\right) W(x, \omega) \mathrm{d} \omega \\
& -\log \left|B_{\mathrm{p}}(x)\right| \\
\log \left(\gamma_{\mathrm{opt}}\right) \geq & \log \left|S^{\prime}(x)\right| \\
& +\frac{1}{2 \pi} \int_{0}^{\infty} \log \left(1+|G(j \omega)|^{2}\right) W(x, \omega) \mathrm{d} \omega \\
& -\log \left|B_{\mathrm{p}}(x)\right| .
\end{aligned}
$$

(iii) The sensitivity function $S_{x}^{\prime}(s):=(1+$ $\left.G_{x}(s)\right)^{-1}$ must be considered instead of (25). If $G(s)$ has poles in the open right half-plane, the factorization (26) must be replaced by $S_{x}^{\prime}(s)=$ $S_{0}^{\prime}(s) B_{\mathrm{p}}^{\prime-1}(s) B_{\mathrm{p}}(s)$, where $B_{\mathrm{p}}(s)$ is the Blaschke product of ORHP poles of $G(s)$. Hence, (28) must be replaced by

$$
\begin{aligned}
\log \left|S^{\prime}(x)\right|= & \frac{1}{\pi} \int_{0}^{\infty} \log \left|S^{\prime}(j \omega)\right| W(x, \omega) \mathrm{d} \omega \\
& -\log \left|B_{\mathrm{p}}^{\prime}(x)\right|+\log \left|B_{\mathrm{p}}(x)\right| .
\end{aligned}
$$

(iv) Since $G_{x}(s)$ is chosen so that $G_{x}(x) \geq 0$, it follows that $S_{x}^{\prime}(s)$ has no pole at $x$.

Theorem 5.3 can be applied to find a lower bound on the optimal cost for plants with poles or zeros in the open right half-plane. Even though additional limitations due to such poles 
and zeros do not appear explicitly in (29), they may appear indirectly because such poles and zeros will make it less likely that $\left(1+G_{x}(s)\right)^{-1}$ is stable.

\section{CONCLUSIONS}

In this paper we have shown how well known design limitations in linear time-invariant feedback systems manifest themselves in the design methodology of McFarlane and Glover (1990). We have done so by presenting lower bounds on the optimal cost that depend upon the properties of the desired loop shape. These bounds provide verification of observations in McFarlane and Glover (1990) and may prove useful in selecting a desired loop shape compatible with robust stability against coprime factor uncertainty.

\section{REFERENCES}

Christian, L. L. and J. S. Freudenberg (1993). Limits on achievable robustness against coprime factor uncertainty.
In Proc. of the 1993 American Control Conference, San Francisco, $\mathrm{CA}$

Doyle, J. C., B. A. Francis and A. R. Tannenbaum (1992). Feedback Control Theory. Macmillan Pub. Co., NY.

Freudenberg, J. S. and D. P. Looze (1985). Right half-plane poles and zeros and design tradeoffs in feedback systems. IEEE Trans. Automat. Control, 30, 555-565.

Freudenberg, J. S. and D. P. Looze (1988). Frequency Domain Properties of Scalar and Multivariable Feedback Systems, Vol. 104. Lecture Notes in Control and Information Sciences, Springer-Verlag, NY.

Georgiou, T. T. and M. C. Smith (1990). Optimal robustness in the gap metric. IEEE Trans. Automat. Control, 35, $673-685$.

Glover, K. and D. C. McFarlane (1989). Robust stabilization of normalized coprime factor plant descriptions with $\mathrm{H}_{x}$-bounded uncertainty. IEEE Trans. Automat. Control, 34, 821-830.

Looze, D. P. and J. S. Freudenberg (1991). Limitations of feedback properties imposed by open-loop right-half plane poles. IEEE Trans. Automat. Control, 36, 736-739.

McFarlane, D. C. and K. Glover (1990). Robust Controller Design Using Normalized Coprime Factor Plant Descriptions, Vol. 138. Lecture Notes in Control and Information Sciences, Springer-Verlag, NY.

Vidyasagar, M. and H. Kimura (1986) Robust controllers for uncertain linear multivariable systems. Automatica, 22, 85-94. 